Stocker, B. A. D. (1955). J. gen. Microbiol. 12, 375-381

\title{
Bacteriophage and Bacterial Classification
}

\author{
By B. A. D. STOCKER \\ Lister Institute of Preventive Medicine, London, S.W. 1
}

Burnet (1933), and more recently Adams (1952), have discussed the classification of certain phages, and have shown that some of their characters are highly correlated with each other, and are therefore criteria of value in the classification of phages; the value of such a classification is not diminished by our ignorance of the evolutionary origins of the phages.

Bacteriophage is of interest in the study of the classification of bacteria. A particular phage will attack and lyse only a characteristic, limited, range of bacterial strains, and such susceptible strains, able to support the multiplication of a particular phage, are always more or less closely related to one another. So that to show that two bacterial strains are both sensitive to a single phage may give information of value as to their taxonomic relationship.

Phages show varying degrees of specificity, ranging from those which seem to attack but a single strain, to others which attack even strains of different genera. Phages which attack some, but not all, strains of a species have been extensively used for phage-typing; I shall not discuss these phages, but shall consider phages that are of value in defining a bacterial species, genus or larger group.

In the case of a species which is already fairly well defined by several correlated characters, the testing of a number of phages on a group of strains of that species and of related species often shows the existence of 'speciesspecific' phages, that is phages which attack all (or nearly all) strains of one species, and no strains of any other species. The existence of such phages is evidence of the value of the criteria used to define the species. Such speciesspecific phages have been used for the routine identification of strains from clinical material, and Katznelson \& Sutton (1951) have shown that by their use it is even possible to test for the presence of a particular bacterium in a sample without prior isolation of strains. They were concerned with testing seeds for the presence of the plant pathogen Pseudomonas phaseolicola, a bacterium for which they had isolated a species-specific phage; a small amount of this phage was added to a mash of the seeds to be examined; an increase in the titre of the phage on incubation showed that the host bacterium was present. Species-specific phages would probably be more widely used in routine bacteriology if there were some central collection from which they could easily be obtained.

In the more fundamental problem of defining a bacterial species, phages may be of great value. For instance, Smith, Gordon \& Clark (1952), in their work on the aerobic mesophilic spore-bearers, examined a large number of spore-bearers, using many different biochemical and other tests, and also 
determined the sensitivity of their strains to various phages isolated from soil. They were able to redefine certain species in terms of bacterial characters that were highly correlated with each other, and they found that some of their phages were specific for the species thus delimited. For instance, one phage attacked all of 145 strains of Bacillus cereus, another attacked all of 70 strains of $B$. pumilus and a third attacked $83 \%$ of 90 strains of B. megaterium; so far as they were tested none of these phages attacked any strains belonging to related species.

A bacterial character is of value as a classificatory criterion to the extent that it correlates with other characters; and in the work of Smith et al. the high correlation of phage sensitivity with other characters (e.g. biochemical and morphological) showed the value of both phage reaction and these other characters in the classification of the genus Bacillus.

The bacterial character likely to be of use to the taxonomist is the susceptibility of the bacterium to lysis by the phage (or the ability of the bacterium to form a lysogenic system with the phage). The mere absorption of a phage is not likely to be a good criterion, for it is known that some phages may be non-specifically absorbed, e.g. by glass under certain conditions; also, particular antigens, which probably form the point of primary absorption, are known to be widely distributed, so that a certain antigen may recur in two bacterial species which are by no means closely related. Rakieten \& Rakieten (1937) found that a staphylococcus phage was absorbed by a strain of Bacillus subtilis, though the latter was not lysed by the phage; they found some evidence for the existence of a common antigen in the two strains.

A phage-sensitive strain can commonly become resistant to a phage by a single mutational step; or it may in some cases acquire resistance by becoming lysogenic. It is, therefore, not surprising to find that phages which attack only strains of a single species often do not attack every strain of that species. Using such a phage, sensitivity identifies an unknown strain as belonging to the species; but resistance does not exclude the identification. That is, only a positive result is of value, as is the case with many other tests, such as the ability of various species to produce characteristic toxins or antigens.

In addition to species-specific phages there have been reported phages which are specific for groups of higher order, such as those we arbitrarily designate as genera. Of greater interest, however, are the phages with an even wider range, since they give indications of the relationships of different genera, a matter which is generally more in dispute than those of species. In the early phage literature there are a number of reports of the 'adaptation' of, for instance, corynebacterium and staphylococcus phages to attack organisms of the Shigella group, and so on. However, this early work now seems unconvincing since the experiments did not prove that the 'adapted' phage was in fact derived from the original phage; in the absence of appropriate controls it now seems impossible to be sure that the 'adapted' phage was not a mutant of a phage carried by the new host, or even a laboratory contaminant. I have not been able to find in the literature any indubitable instances of phages 
which attack strains not belonging to what I should consider the same family.

The grouping together of the genera Escherichia, Salmonella and Shigella in the family Enterobacteriaceae is confirmed by the reports of numerous phages which attack strains from two or all three of these genera. It is well established that some phages which attack both Pasteurella pestis and $P$.pseudotuberculosis also attack some Shigella and Escherichia strains; and some phages originally described as Shigella phages, such as Burnet's phage C16, also attack $\boldsymbol{P}$. pestis (Flu, 1927; Girard, 1943; Flu \& Flu, 1946; Lazarus \& Gunnison, 1947). To me this indicates fairly close relationship, and suggests that $P$. pestis and $P$.pseudotuberculosis should be included in the family Enterobacteriaceae, and not relegated to a heterogeneous family such as the Parvobacteriaceae of the 6th edition of Bergey's Manual; indeed if one compares the morphology, biochemical activities and nutritional requirements of these two species with those of the Shigella group it is hard to find any good differentiating criteria. On the other hand, there do not seem to be any phages reported which attack both $P$. septica and also $P$. pestis or $P$. pseudotuberculosis; this casts some doubt on the propriety of placing $P$. septica in the same genus as the other two species, a grouping which has been criticized on other grounds (Girard, 1942).

Though phages whose range encompasses several species of Enterobacteriaceae are common, none of them seems to be known to attack Proteus strains; little has been reported about proteus phages, but there is no good evidence that they attack anything but Proteus spp. This suggests that Proteus is less closely related to Escherichia, Shigella and Salmonella than these latter are related to each other. Here, too, it seems to me that the hint given by the phages is confirmed by other considerations; for Proteus, or at any rate $\boldsymbol{P}$. mirabilis and $\boldsymbol{P}$. vulgaris, differ from the typical enterobacters in quite a number of characters, such as their ability to swarm, their very poor growth anaerobically, their high urease activity, their production of phenylpyruvic acid from phenylalanine, their high resistance to many antibiotics and other antibacterial agents and their production of certain amines.

Some effects of phage other than lysis are also of interest to the taxonomist. In the transduction of characters from one strain of Salmonella to another it now seems clear that the role of phage is merely that of a vector of host genetic material from one cell to another. The process is essentially equivalent to the transformations of pneumococci, meningococci and Haemophilus influenzae, where deoxyribonucleic acid in the free form is effective, no vector being needed. The transformed bacterium, for instance a strain of Salmonella typhi which has been given the flagellar antigen $i$ of $S$. typhimurium, is in effect a hybrid of two strains, but a hybrid which has derived all but one of its characters from one of the two parents. Our ability to hybridize two bacterial strains in the laboratory has no more (and no fewer) implications for taxonomy than has the artificial hybridization of two higher plants or animals. It does not invalidate a classification which assigns the two parents to separate groups, e.g. species, but it does suggest that the parental strains are fairly 
closely related. There is, as yet, no evidence that these or other phenomena of bacterial hybridization occur under natural conditions. It is, therefore, still possible to treat bacteria as organisms which in nature multiply only by fission, without a sexual process or other mechanism of gene interchange; we may then consider all strains of a valid species, genus or larger taxonomic group as members of a clone, all derived from a single common ancestor. If we assume this we may regard any good practical classification (that is one based on multiple correlated characters) as being of necessity an approximation to a phylogenetic classification.

Many of the bacterial strains which we have to classify are lysogenic; indeed in some species it seems probable that all strains are lysogenic. In such species the characters which we record are not those of the bacterium alone, but those of a stable complex of bacterium and phage (in the latent or prophage form). There is some resemblance to the case of the lichens, which were classified before it was known that each was a symbiotic system comprising an alga and a fungus. The presence of latent phage or prophage in a bacterium does not in general seem to produce very striking effects on bacterial characters such as biochemical activities or antigenic constitution. But the recent work on phage-induced toxigenicity in Corynebacterium diphtheriae seems to indicate that here the presence of a particular phage in a bacterium causes it to produce toxin, a character of great interest to the taxonomist. Ideally it would be desirable to study the characters of both the bacterium in its original lysogenic state, and after it had ceased to be lysogenic; but usually we have no means of freeing a lysogenic strain of its phage. However, as the lysogenic complex is in many species the modal form, and that which has been moulded by evolutionary forces, our inability to study the non-lysogenic form does not much reduce the value of our present system of classification, nor invalidate it as a representation of natural relationships.

\section{REFERENCES}

Adams, M. H. (1952). Classification of bacterial viruses; characteristics of the T5 species and of the T2, $\mathrm{C} 16$ species. J. Bact. 64, 387 .

Burnet, F.M. (1933). The classification of dysentery-coli bacteriophages. III. Acorrelation of the serological classification with certain biochemical tests. J. Path. Bact. 37, 179.

Flu, P. (1927). Sur la nature du bactériophage. C.R. Soc. Biol., Paris, 96, 1148.

Fuv, P. \& FuU, H. (1946). On bacteriophages against plague, occurring in canal water and sewage in the Netherlands, and of the origin of these phages. Leeuroenhoek ned. Tijdschr. 11, 195.

Girard, G. (1942). Sur quelques nouveaux caractères différenciant les bacilles de la peste et de la pseudotuberculose des Pasteurella. Ann. Inst. Pasteur, 68, 476.

Grand, G. (1943). Sensibilité des bacilles pesteux et pseudotuberculeux d'une part des germes du groupe coli-dysentérique d'autre part aux bactériophages homologues. Ann. Inst. Pasteur, 69, 52.

Katznelson, H. \& Sutton, M. D. (1951). A rapid plaque count method for the detection of bacteria as applied to the demonstration of internally borne bacterial infections of seed. J. Bact. 61, 689 . 
Lazarus, A. S. \& Gunnison, J. B. (1947). The action of Pasteurella pestis bacteriophage on strains of Pasteurella, Salmonella and Shigella. J. Bact. 53, 705.

Rakieten, M. L. \& Rakieten, T. L. (1937). Relationships between staphylococei and bacilli belonging to the subtilis group as shown by bacteriophage absorption. J. Bact. 34, 285.

Smith, N. R., Gordon, R. E. \& ClakK, F. E. (1952). Aerobic sporeforming bacteria. U.S. Dep. Agric., Monograph no. 16.

\section{DISCUSSION}

By E. S. Anderson, Central Enteric Reference Laboratory, Colindale Avenue, London, N.W. 9

Identification and grouping by phage is an additional tool to be used in classification in association with the other information we can obtain about an organism.

There are clear-cut patterns exhibited by Salmonella paratyphi-B and $S$. typhimurium with the typing phages of Felix \& Callow $(1943,1951)$ that appear to be specific for each serotype and are not shown by any other salmonellas with these sets of phages. The use of patterns is essential because the phages employed are not completely specific for either $S$. paratyphi-B or $S$. typhimurium but will attack other members of the $\mathbf{B}$ group or even members of other somatic groups. These patterns, which are an expression of the resistance or sensitivity of the organisms to groups of phages, frequently owe their form to lysogenicity of the strains being examined.

If an organism shows the lytic pattern of a recognized phage type of Salmonella paratyphi-B it can be safely accepted as being $S$. paratyphi-B whether it is monophasic or even non-motile. We have encountered a strain with the antigenic formula $4,5,12: b-$, and the biochemical characteristics of $S$. paratyphi-B, from clinical cases of enteric fever. This strain is obstinately monophasic and, lacking a second flagellar phase, cannot be designated as $S$. paratyphi-B by the serodiagnostic scheme. Bacteriophage typing identifies it as phage type Jersey of the paratyphoid-B bacillus, a monophasic strain of that serotype.

In the same way, monophasic and non-motile strains having the somatic formula 4, 5, 12:-1, 2, 3 and 4, 5, 12 :- have been identified as Salmonella typhimurium. In one instance, a non-motile variant identified as $S$. typhimurium phage type 4 was later found to be connected with an outbreak caused by a motile strain of the same phage type.

Another example of the use of phage in classification is found in the Viphages of the typhoid bacillus (Craigie \& Yen, 1938). Vi-phage II, the typing phage, is capable of modification to a minimum of thirty-three different specificities; these constitute the typing phages. According to the lytic pattern they produce on a strain of Salmonella typhi, the Vi-typing phages tell us a great deal about the organisms; we can say whether the strain is lysogenic and it is even possible to describe the characteristics of the phage it carries (Anderson \& Felix, 1953; Anderson, to be published). 
Strains of Salmonella paratyphi-C, paracolon bacilli and Escherichia coli possessing the Vi antigen are sensitive to Craigie \& Yen's Vi-phages (Nicolle, Rita \& Huet, 1951) but require higher concentrations to produce confluent lysis than does $S$. typhi.

The principle of identifying regular lytic patterns-the so-called "phage types'-in the paratyphoid-B bacillus and Salmonella typhimurium can be extended to other salmonellas, as has been shown by Williams Smith $(1951 a, b)$ with $S$. dublin and $S$. thompson. Once these patterns are established with certainty, the method can be inverted to identify the serotype by the pattern of phage lysis.

Certain characters of some salmonella serotypes are regularly associated with definite phage types. There is, for example, a phage type of Salmonella typhimurium that is always anaerogenic. This type was first encountered in 1943 and is still causing outbreaks in different parts of the country.

The only way in which Salmonella paratyphi-B can be distinguished from $S$. typhimurium antigenically is by flagellar serology. The phages that effect this distinction, however, do not attack the flagella; their substrate is entirely the soma of the organism. The reasons for the difference in host specificity of these closely related pathogens-man with paratyphoid-B, and rodents with typhimurium-are unknown, but they are certainly a function of the somatic make-up of the organisms, and it is evident that the phages are capable of recognizing somatic differences that are inapparent to the reticulo-endothelial system of the rabbit.

\section{REFERENCES}

Anderson, E. S. \& Felux, A. (1953). 'Degraded Vi strains' and variation in Viphage II of Salmonella typhi. J. gen. Microbiol. 8, 408.

Craigie, J. \& Yen, C. H. (1938). The demonstration of types of B. typhosus by means of preparations of Type II Vi phage. Canad. publ. Hlth J. 29, 448, 484 .

Felix, A. \& Callow, B. R. (1943). Typing of paratyphoid-B bacilli by means of Vi bacteriophage. Brit. med. J. ii, 127.

Felix, A. \& Callow, B. R. (1951). Paratyphoid-B Vi-phage typing. Lancet, ii, 10.

Nicolle, P., Rita, G. \& Huet, M. (1951). Démonstration, par l'épreuve des bactériophages $\mathrm{Vi}$, d'une analogie physiologique entre les antigènes Vi présents chez des espèces bactériennes différentes. Ann. Inst. Pasteur, 81, 473.

Williams Smith, H. (1951 $a)$. The typing of Salmonella thompson by means of bacteriophage. J. gen. Microbiol. 5, 472.

Williams Smith, H. (1951 b). The typing of Salmonella dublin by means of bacteriophage. J. gen. Microbiol. 5, 919.

\section{DISCUSSION}

\section{By A. Feurx, Lister Institute, London}

I congratulate Dr Stocker on his refreshing and original presentation of the subject. I should also like to congratulate the organizers of the symposium on their bold decision to place bacteriophage on the agenda separately from the item 'The classification of viruses'. Dr Stocker did not speak about classifica- 
tion of phages but showed that phages can be employed as a good taxonomic agent for the classification of bacteria, leading to a natural phylogenetic system of bacteria. In my view this meant treating phage as an integral part of the genetic make-up of the bacterium, not as a parasite of extrinsic origin as postulated for animal viruses. Dr Stocker very rightly refrained from discussing 'phage-typing'. This is a technique of bacterial identification for a specific purpose, namely epidemiology, and has little to do with bacterial classification. A point Dr Stocker might perhaps have mentioned is the relationship between colicines and phages. 